\title{
Autoestima e comportamentos de saúde bucal em adolescentes
}

\author{
Self-esteem and oral health behavior in adolescents
}

Carolina Thaiza Costa Pazos (http://orcid.org/0000-0002-0348-3461) ${ }^{1}$

Silvia Carréra Austregésilo (https://orcid.org/0000-0002-1187-8068) ${ }^{1}$

Paulo S. A. de Goes (https://orcid.org/0000-0002-6708-0450) ${ }^{2}$

${ }^{1}$ Programa de PósGraduação em Saúde da Criança e do Adolescente, Universidade Federal de Pernambuco (UFPE). R. Prof. Pedro Augusto Carneiro Leão 585, BL E-10, Apt 103, Imbiribeira. Recife PE Brasil.

carolthaiza@gmail.com

${ }^{2}$ Departamento de Odontologia Clínica e Preventiva, Universidade Federal de Pernambuco (UFPE). Recife PE Brasil.

\begin{abstract}
This study investigated the mediation of self-esteem in adolescents' oral health behaviors. The Rosenberg self-esteem scale was used to assess self-esteem, whereas data from sociodemographic and behavior characteristics were analyzed by questions validated in previous surveys. The teenagers had good oral health behavior, except unhealthy diet. The number of adolescents with high self-esteem was a lot smaller than those with low self-esteem. The use of dental services, even when associated with high self-esteem, lost significance after being adjusted by sex, age and tooth brushing frequency. Nevertheless, multiple logistic regression analysis, using unadjusted estimates and adjusted with their respective Confidence Intervals of 95\%, showed a relationship of self-esteem with age $(p$-value $=0.001)$ and tooth brushing frequency ( $p$-value=0.019). Regardless of the sex, students over 16 years old with high self -esteem brush their teeth more often, having probably better oral health. These results confirm the modulation of self-esteem in oral health, and then it is necessary the analysis and the use of these psychosocial factors in the young oral health care. Keywords Self-esteem, Health behavior, Oral health, Adolescent.
\end{abstract}

Resumo Este estudo investigou a mediação da autoestima nos comportamentos de saúde bucal dos adolescentes. Para avaliar a autoestima usouse a escala de Rosenberg, já as características sociodemográficas e comportamentais foram analisadas por questões validadas em outros inquéritos. Com exceção da alimentação, a maior proporção dos adolescentes possuía bons comportamentos de saúde bucal. O número de adolescentes com alto nivel de autoestima foi relevantemente menor que aqueles com baixa autoestima. A utilização dos serviços odontológicos, embora associado ao alto nível de autoestima, perdeu significância após ajustado pelo sexo, idade e escovação dos dentes. Porém, a análise de regressão logística múltipla, através das estimativas não-ajustadas e ajustadas com seus respectivos Intervalos de Confiança de 95\%, evidenciou associação da autoestima com idade ( $p$-valor $=0,001)$ e frequência de escovação ( $p$-valor=0,019). Independente do sexo, escolares maiores de 16 anos e com autoestima elevada, escovam seus dentes com maior frequência, adquirindo, possivelmente, melhor saúde bucal. Isto confirma a modulação da autoestima sobre os comportamentos de saúde bucal, e atesta a necessidade de implementar a análise e o exercício desse fator psicossocial na assistência à saúde bucal dos jovens.

Palavras-chave Autoestima, Comportamentos saudáveis, Saúde bucal, Adolescente. 


\section{Introdução}

O gradiente existente na relação entre os comprometimentos de saúde e os fatores socioeconômicos foi estabelecido, no fim do século passado, por Marmot et al. ${ }^{1}$ ao enfatizar a importância da maneira de como as pessoas tinham o controle de suas vidas nos desfechos de saúde. Ou seja, as doenças e os comportamentos de saúde não são consequências apenas das circunstâncias econômicas da função trabalhista exercida. Mas, poder de decisão, ambiente de trabalho, controle, satisfação e apoio social são mediadores dos comportamentos e condições de saúde ${ }^{1-4}$.

Embora existam muitos trabalhos de saúde bucal que tentam investigar as condições de saúde bucal a partir dos comportamentos, são poucos os estudos que trabalham os fatores psicossociais, e são menos ainda aqueles que trabalham em populações homogêneas. Assim, visamos elucidar se os fatores psicossociais são responsáveis pelas diferenças nos comportamentos de saúde bucal de uma população de escolares socioeconomicamente homogêneos.

$\mathrm{Na}$ adolescência, intensas modificações biopsicossociais inserem os adolescentes em um dos grupos com maior vulnerabilidade aos agravos sociais $^{5-8}$ e de saúde ${ }^{8,9}$. Hábitos adquiridos nesse período repercutem em dimensões futuras, como alimentação, autoimagem, saúde individual, valores, preferências e desenvolvimento psicossocial ${ }^{10}$.

Nessa fase, os momentos de negligência com os cuidados à saúde tornam-se comuns. Enquanto os comportamentos que contribuem para manutenção da saúde bucal são reduzidos ${ }^{11}$, a prevalência do consumo de álcool e fumo torna-se alarmante e com repercussões na saúde desses jovens ${ }^{8,11-14}$.

Estudos têm destacado a associação entre os hábitos de saúde, como os de higiene bucal ${ }^{15,16}$ e geral ${ }^{17}$, utilização de serviços odontológicos ${ }^{18}$, uso de drogas, cigarro e bebidas alcoólicas ${ }^{19}$, com os fatores psicossociais ${ }^{17,20}$. Dentre esses, a autoestima pode ter relação com as práticas de saúde e mecanismos de estresse, que expõem os adolescentes ao aumento de riscos físicos e distúrbios psicológicos ${ }^{21-23}$.

Devido às mudanças ocasionadas pela adolescência, esta é a fase na qual a autoestima assume as proporções mais expressivas, com consequências que podem alterar os comportamentos de saúde por toda vida do indivíduo. Fato que torna a autoestima um importante preditor de resultados nos estudos em adolescentes e adultos joven ${ }^{24}$.
Além disso, apesar de algumas investigações indicarem que a autoestima elevada está associada à satisfação com a vida, menos problemas de saúde, escovação e visitas ao dentista mais frequentes e menos impactos na saúde bucal dos adolescentes ${ }^{21,25}$, e que a autoestima instável aumenta os riscos para saúde bucal ${ }^{26}$, são raras e inconsistentes as explicações apontadas pelos estudos quando procura-se conhecer associações entre a autoestima e os comportamentos de saúde bucal entre os adolescentes.

Diante do exposto, da hipótese que os fatores psicossociais influenciam a saúde, e da escassez de pesquisas sobre o tema em saúde bucal com uma população socioeconomicamente homogênea, o presente trabalho se propõe investigar a mediação da autoestima nos comportamentos de saúde bucal dos adolescentes, em função de suas características sociodemográficas.

\section{Método}

A presente pesquisa é definida como estudo de corte transversal, analítico, com abordagem quantitativa e faz parte da segunda fase do levantamento epidemiológico "Associação entre fatores psicossociais e saúde bucal", realizado em 2014, com adolescentes de 14 a 19 anos matriculados em escolas públicas do Município de São Lourenço da Mata - Pernambuco.

A cidade de São Lourenço da Mata foi selecionada por ter se constituído pólo de desenvolvimento da Região Metropolitana do Recife a partir da instalação do Complexo da Arena Pernambuco e grandes investimentos imobiliários, os quais poderão repercutir nas condições de vida da população adolescente.

Considerado uma das cidades mais antigas do Brasil, o município está localizado a $16 \mathrm{~km}$ da capital pernambucana, possui área de $262 \mathrm{~km}^{2} \mathrm{e}$ densidade demográfica acima dos 392 habitantes $/ \mathrm{km}^{2}$. Sendo $92 \%$ dos seus 102.895 habitantes residente em zona urbana.

Entre os dados da economia de São Lourenço da Mata destacam-se o crescimento do setor de serviços e indústrias, o Produto Interno Bruto (PIB) per capita de R \$5.369,34 e o Índice de Desenvolvimento Humano (IDH) do município em média de $0,653^{27}$.

O cálculo amostral foi realizado considerando os desfechos de saúde bucal investigados em estudo anterior ${ }^{28}$ e no levantamento epidemiológico ao qual o presente trabalho está aninhado ${ }^{29-32}$. Dentre esses desfechos a dor de origem dentária 
apresentou a menor prevalência, 20\%. Assim, por definição estatística, tudo que for $>20 \%$ está representado neste conjunto.

Dessa maneira para os cálculos do tamanho amostral, no estudo como um todo, foi utilizada a fórmula de comparação de duas proporções, relação de 1:1 entre os grupos, com uma prevalência esperada de $20 \%$ de dor de dente nesta população, poder de teste de $80 \%$, erro aleatório de $2,5 \%$ e intervalo de confiança (IC) de $95 \%$. Foram utilizados o programa de cálculo do Epi Info 6.

Optamos trabalhar com adolescentes de escola pública para obter uma amostra considerada homogênea do ponto de vista socioeconômico. De acordo com dado fornecido pela Secretaria de Educação do Município, no período da coleta, a rede pública de educação contava com 49 instituições de ensino municipais (entre escolas e creches) e oito escolas estaduais. O quantitativo de alunos matriculados nos anos finais do ensino fundamental e no ensino médio somavam 8.393 na rede pública e 1.464 na rede privada ${ }^{31}$.

Participaram da pesquisa 11 escolas públicas que possuíam alunos da faixa etária pretendida pelo estudo, sendo composta por uma amostra de 1.154 estudantes, representando uma taxa de resposta de $81,5 \%$ da amostra inicialmente calculada. Cada escola contribuiu de forma proporcional ao número de matriculados na idade da pesquisa, estabelecendo-se desta forma um quociente de proporcionalidade.

Foi realizado um sorteio randomizado, a partir da lista nominal de escolares, os quais foram sorteados a partir do primeiro nome da lista, alternando-se um adolescente selecionado com um não selecionado, excluindo-se o $12^{\circ}$ nome. Foram excluídos do estudo os indivíduos com dificuldade de compreensão para responder ao questionário.

Para a coleta dos dados foi utilizado um questionário autoaplicável, constituído por escalas com validade e utilizadas em outros grandes inquéritos ${ }^{33-35}$, que aconteceu nas próprias dependências das escolas, após prévia explicação dos objetivos e métodos do estudo, sendo retiradas todas as dúvidas que surgissem no momento da pesquisa. Com o objetivo de realizar o controle de qualidade dos dados, a aplicação dos questionários foi refeita a cada dez participantes. Os resultados demonstraram um grau aceitável para as análises de reteste $(r>0,8)$.

Como variável dependente foi selecionada o fator psicossocial, mensurado a partir da análise do componente "Autoestima", a qual foi avaliada segundo a escala de Rosenberg ${ }^{32}$, que se refere à posição do adolescente quanto ao apreço ou valorização que cada um tem de si próprio, mediante situações adversas ou não, permitindo-lhe confiança sobre suas ações.

Essa escala é considerada medida padrão, em função de suas boas propriedades psicométricas e fácil aplicabilidade. A versão aqui utilizada foi a proposta por Sbicigo et al. ${ }^{33}$, traduzida para o português, devido a boa consistência interna. Trata-se de uma escala de três pontos do tipo Likert, para facilitar a compreensão dos adolescentes $(1=$ discordo; $2=$ nem concordo, nem discordo; $3=$ concordo), com 10 questões, das quais seis avaliam sentimentos positivos do indivíduo sobre si mesmo (de modo geral, estou satisfeito comigo mesmo; eu acho que tenho muitas boas qualidades; eu sou capaz de fazer tudo tão bem como as outras pessoas; eu sinto que sou uma pessoa de valor como as outras pessoas; eu tenho uma atitude positiva em relação a mim mesmo; eu tenho motivos para me orgulhar na vida) e quatro referem visão depreciativa (às vezes, eu penso que não presto para nada; eu sinto vergonha do jeito que sou; às vezes, eu me sinto inútil; levando tudo em conta, eu me sinto um fracasso $)^{33}$.

Diante da distribuição não normal da variável autoestima, da necessidade de estabelecer uma regra para evidenciar o fator de risco do estudo, e com base no conhecimento que a dicotomização de variáveis categóricas é aleatória de acordo com estatística, mesmo com o risco de perder detalhes, para a análise foi criada uma variável binária a partir da utilização do percentil $75 \%$, sendo considerado como alto nível de autoestima aqueles com valor geral da escala acima deste percentil.

As variáveis independentes foram ordenadas em dois grupos: o primeiro referia-se aos dados sociodemográficos (sexo, idade, histórico de reprovação, estrutura familiar e ordem de nascimento entre os irmãos); e o segundo referia-se aos comportamentos de saúde bucal (utilização de serviços, hábitos de higiene, alimentação saudável, consumo de cigarro e álcool).

Os comportamentos em saúde também foram dicotomizados, baseado nos padrões favoráveis de saúde e nos parâmetros utilizados em pesquisas anteriores ${ }^{8,18,36}$, para a análise da influência da autoestima nesses hábitos, como descrito no Quadro 1. Especificamente nas variáveis utilização do serviço odontológico, hábitos de higiene bucal e hábitos alimentares, o adolescente precisava se enquadrar em pelo menos um item para ser classificado em determinada categoria. 


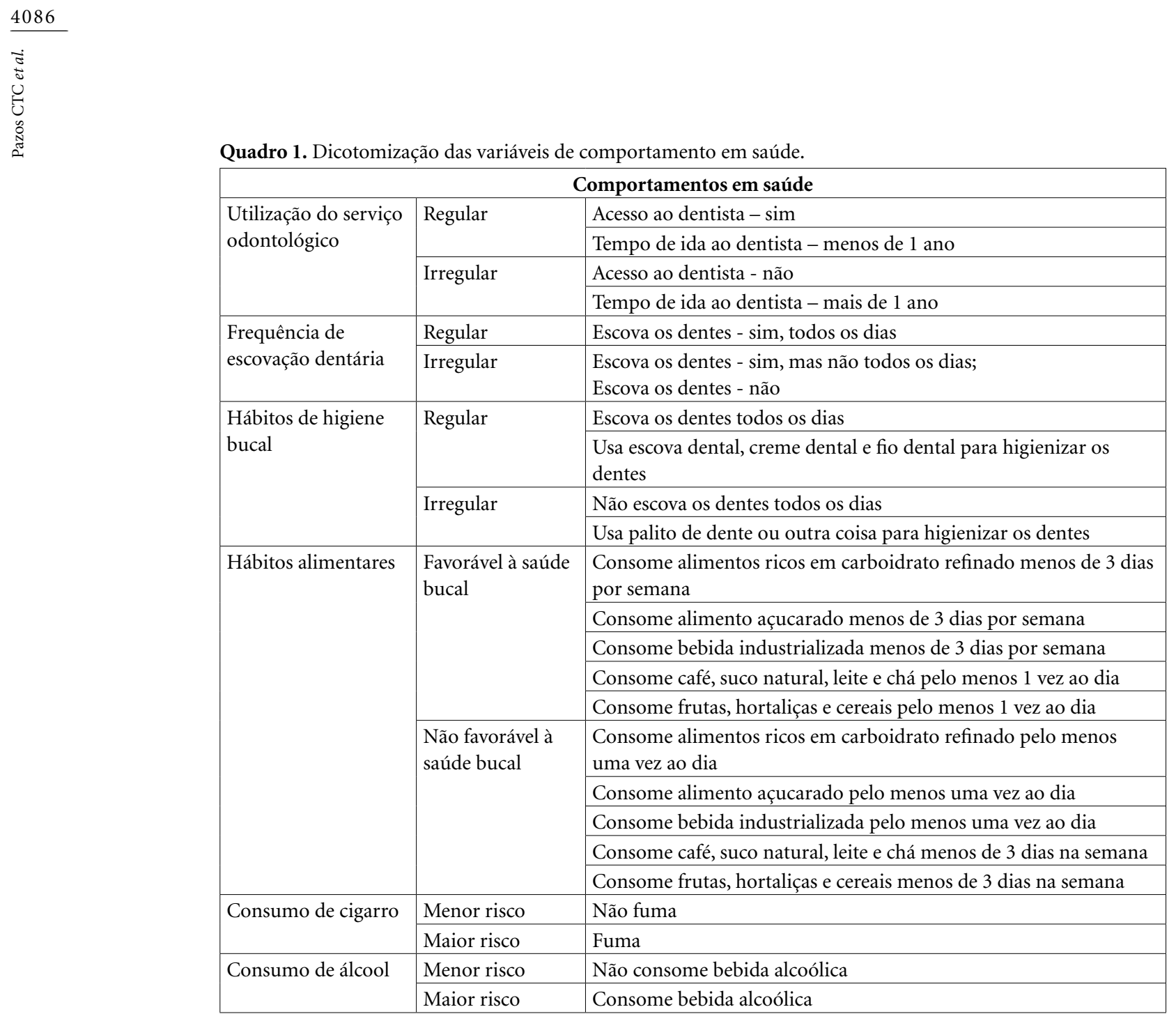

Essa medida foi adotada porque as variáveis independentes eram representadas por muitos itens. Assim, criamos variáveis de contagem, avaliamos as medidas de tendência central e dispersão, e testamos a normalidade através do teste de Kolmogorov-Smirnov. Como a normalidade não foi provada e os testes logarítmicos e quadrados não ficaram adequados, optamos pela categorização a partir da mediana, por ser a medida mais estável do ponto de vista aritmético. Os cálculos amostrais das variáveis dependente e independentes estudadas foram fundamentados ao encontrado em outra pesquisa ${ }^{28}$.

A análise dos dados foi realizada utilizado-se o programa Statistical Package for the Social Sciences (SPSS), versão 18.0 (SPSS para Windows, versão 18.0, EUA). Foram calculadas as frequências percentuais e construídas as respectivas distribuições de frequência dos dados sociodemográficos e comportamentais. Ainda, foram calculadas as prevalências do nível de autoestima dos alunos avaliados.

Para avaliar a influência da autoestima nos comportamentos de saúde bucal dos alunos foi aplicado o teste Qui-quadrado para independência. Nos casos em que as suposições do teste Qui-quadrado não foram satisfatórias, aplicou-se o teste Exato de Fisher. Todas as conclusões foram extraídas considerando o nível de significância de 5\%.

$\mathrm{Na}$ análise de regressão logística múltipla, apenas foram consideradas as variáveis que apresentaram significância na análise bivariada, a exceção do sexo. Esta foi mantida devido sua importância demonstrada pelo modelo teórico. As variáveis entraram no modelo por blocos, pelo método ENTER e a consistência dos modelos foi avaliada pelo teste de Hosmer-Lemeshow, sendo apresentadas as estimativas não-ajustadas e ajustadas com seus respectivos IC de 95\%. O rigor na 
entrada das variáveis para essa análise e a preocupação em realizar o ajuste foram tomados para minimizar o efeito de superestimar, da regressão logística.

A pesquisa foi conduzida de acordo com os princípios éticos, em consonância com a Resolução n 466/2012 do Conselho Nacional de Saúde - CNS, e foi aprovada pelo Comitê de Ética em Pesquisa da Universidade Federal de Pernambuco (CEP/UFPE). Os escolares participaram da pesquisa mediante assinatura do Termo de Consentimento Livre e Esclarecido (TCLE), por aqueles com idade de 18 ou 19 anos. Quando menores de idade, foi solicitada a assinatura do Termo de Assentimento Livre e Esclarecido, além da assinatura do TCLE pelo responsável.

\section{Resultados}

Para esta pesquisa, dos 1.154 adolescentes participantes do estudo, com idades entre 14 e 19 anos, verificou-se que a maior proporção é do sexo feminino $(53,5 \%)$, tem menos de 16 anos de idade $(52,1 \%)$, nunca experimentou uma reprovação escolar $(56,1 \%)$, mora em família com núcleo familiar $(55,1 \%)$ e é o primeiro na ordem de nascimento de sua família (45,3\%).

Em relação aos fatores comportamentais de saúde bucal, verifica-se que a maioria dos alunos escovam os dentes todos os dias $(96,6 \%)$, tem acesso à assistência odontológica (92,9\%), foi ao dentista há menos de um ano (54,2\%), sendo a possibilidade do dente estar cariado $(48,4 \%)$ ou escurecido $(39,9 \%)$ as principais razões dessas consultas. Dentre os itens mais utilizados para higiene bucal, está a escova dental $(96,5 \%)$, seguido do creme dental $(83,5 \%)$ e fio dental $(50,8 \%)$. Também se constatou que a maior proporção dos alunos não apresenta boa alimentação no que se refere ao consumo de lanches, doces, bebidas industrializadas, café/leite, chás e frutas/legumes $(96,0 \%)$. No entanto, nota-se que a maioria dos alunos não fumam $(98,7 \%)$ e dos que utilizam cigarro o faz apenas $1 \mathrm{vez}$ ao dia (40,0\%). Assim como, entre os $6,4 \%$ que fazem uso do álcool tem contato apenas uma vez na semana $(88,1 \%)$.

Sobre o nível de autoestima, observamos que a prevalência dos adolescentes com alta autoestima foi menor $(24,1 \%)$ quando comparados aos com baixa autoestima. A distribuição da classificação do nível de autoestima, segundo os fatores sociodemográficos dos adolescentes entrevistados é apresentada na Tabela 1. Destaca-se que, dentre esses fatores, apenas a idade apresentou significância ( $\mathrm{p}$-valor $<0,001$ ), mesmo ao constatar menor proporção da associação entre os fatores sociodemográficos e o alto nível de autoestima.

A distribuição dos comportamentos de saúde bucal segundo o nível de autoestima está descrita na Tabela 2. Verifica-se que o nível de autoestima foi significativo apenas no fator frequência de escovação dos dentes $(p$-valor $=0,004)$ e utilização de serviços odontológicos ( $\mathrm{p}$-valor $=0,033$ ). Ainda se observa que no grupo com baixo nível de autoestima, houve maior prevalência de alunos que não escovam os dentes todos os dias e apresentam maior prevalência da utilização irregular dos serviços odontológicos. Para os demais fatores não foram encontradas diferenças significativas dos hábitos de saúde bucal entre os grupos de baixo e alto nível de autoestima.

Ao empregar no modelo de regressão logística múltipla as variáveis sexo, idade, utilização de serviços e escovação dos dentes, ficou evidenciada, após ajuste, a significância da influência da autoestima na idade ( $\mathrm{p}$-valor $=0,001)$ e na escovação regular $(\mathrm{p}$-valor $=0,019)$, além da perda do valor estatístico da utilização de serviços odontológicos com regularidade $(\mathrm{p}$-valor $=0,110)$ (Tabela 3).

\section{Discussão}

Neste estudo, através de relatos dos escolares de 14 a 19 anos, com características socioeconômicas homogêneas, foi possível estabelecer associação entre autoestima e comportamentos de saúde bucal. Haja vista que adolescentes com padrões mais elevados de autoestima apresentaram comportamentos mais favoráveis à sua saúde bucal, quando comparados aos seus semelhantes com menor autoestima, sendo significante a sua mediação no que se referem à frequência de escovação e à idade. Essa constatação foi possível após a caracterização da população estudada, em relação aos padrões de autoestima, características sociodemográficas e comportamentais relativas à saúde bucal.

Ao considerar as escolas públicas como um indicador de hegemonia socioeconômica, na qual se estabelece uma hierarquia associada aos fatores psicossociais que repercutem nos comportamentos de saúde ${ }^{1-4}$, alertamos para a necessidade de entender o ambiente onde os adolescentes estão inseridos, os fatores psicossociais envolvidos, para então compreender e estimular a adoção de melhores hábitos de saúde bucal. 
Tabela 1. Distribuição da classificação do nível da autoestima segundo os fatores sociodemográficos dos adolescentes matriculados em escolas públicas de São Lourenço da Mata - PE.

\begin{tabular}{lccc}
\hline & Fator avaliado & Nível da autoestima & \multirow{2}{*}{ p-valor $^{\mathbf{a}}$} \\
\cline { 2 - 3 } & Baixo & Alto & \\
\hline Sexo & & & \\
$\quad$ Masculino & $408(77,7 \%)$ & $117(22,3 \%)$ & $\chi^{2}=1,794$ \\
$\quad$ Feminino & $454(74,3 \%)$ & $157(25,7 \%)$ & $\mathrm{p}=0,180$ \\
Idade & & & \\
$<16$ anos & $472(80,3 \%)$ & $116(19,7 \%)$ & $\chi^{2}=13,404$ \\
$\geq 16$ anos & $383(70,9 \%)$ & $157(29,1 \%)$ & $\mathrm{p}<0,001^{\mathrm{b}}$ \\
Histórico de reprovação & & & \\
Sim & $338(73,5 \%)$ & $122(26,5 \%)$ & $\chi^{2}=2,711$ \\
Não & $492(77,6 \%)$ & $142(22,4 \%)$ & $\mathrm{p}=0,258$ \\
Algumas vezes & $27(79,4 \%)$ & $7(20,6 \%)$ & \\
Estrutura familiar & & & \\
Família nuclear & $476(76,3 \%)$ & $148(23,7 \%)$ & $\chi^{2}=0,122$ \\
Família não nuclear & $386(75,4 \%)$ & $126(24,6 \%)$ & $\mathrm{p}=0,727$ \\
Ordem de nascimento & & & \\
Primeiro & $383(75,1 \%)$ & $127(24,9 \%)$ & $\chi^{2}=1,351$ \\
Segundo & $258(76,8 \%)$ & $78(23,2 \%)$ & $\mathrm{p}=0,853$ \\
Terceiro & $119(77,3 \%)$ & $35(22,7 \%)$ & \\
Quarto & $78(76,5 \%)$ & $24(23,5 \%)$ & \\
Não sei & $17(68,0 \%)$ & $8(32,0 \%)$ & \\
\hline
\end{tabular}

${ }^{a}$ p-valor do teste Qui-quadrado para independência.

bSe p-valor $<0,05$ o fator avaliado influencia no nível de autoestima.

Tabela 2. Distribuição dos comportamentos de saúde bucal dicotomizados segundo o nível de autoestima dos adolescentes matriculados em escolas públicas de São Lourenço da Mata - PE.

\begin{tabular}{lccc} 
& \multicolumn{1}{c}{ Fator avaliado } & Nível da autoestima & \multirow{2}{*}{ p-valor } \\
\cline { 2 - 3 } & Baixo & Alto & \\
\hline Escova os Dentes & $814(75,1 \%)$ & $270(24,1 \%)$ & $\mathrm{p}=0,004^{\mathrm{c}}$ \\
Sim, todos os dias & $36(94,7 \%)$ & $2(5,3 \%)$ & \\
$\quad$ Sim, mas não todos os dias & $1(100,0 \%)$ & $0(0,0 \%)$ & \\
$\quad$ Não & & & \\
Hábitos de higiene bucal & $368(73,6 \%)$ & $132(26,4 \%)$ & $\chi^{2}=2,537$ \\
$\quad$ Regular & $494(77,7 \%)$ & $142(33,3 \%)$ & $\mathrm{p}=0,111^{\mathrm{a}}$ \\
$\quad$ Irregular & & & \\
Utilização de serviços odontológicos & $408(73,1 \%)$ & $150(26,9 \%)$ & $\chi^{2}=4,571$ \\
$\quad$ Regular & $454(78,5 \%)$ & $124(21,5 \%)$ & $\mathrm{p}=0,033^{\mathrm{a}, \mathrm{b}}$ \\
$\quad$ Irregular & & & \\
Alimentação & $34(75,6 \%)$ & $11(24,4 \%)$ & $\chi^{2}=0,003$ \\
$\quad$ Favorável à saúde bucal & $828(75,9 \%)$ & $263(24,1 \%)$ & $\mathrm{p}=0,959^{\mathrm{a}}$ \\
$\quad$ Não favorável à saúde bucal & & & \\
Consumo de cigarro & $847(75,8 \%)$ & $271(24,2 \%)$ & $\mathrm{p}=1,000^{\mathrm{c}}$ \\
$\quad$ Menor risco & $12(80,0 \%)$ & $3(20,0 \%)$ & \\
Maior risco & & & \\
Consumo de álcool & $807(76,1 \%)$ & $254(23,9 \%)$ & $\chi^{2}=0,446$ \\
Menor risco & $53(72,6 \%)$ & $20(27,4 \%)$ & $\mathrm{p}=0,504^{\mathrm{a}}$ \\
\hline Maior risco & & & \\
\hline
\end{tabular}

${ }^{\mathrm{a}} \mathrm{p}$-valor do teste Qui-quadrado para independência.

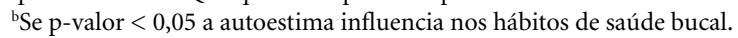

cp-valor do teste Exato de Fisher. 
Tabela 3. Resultados da regressão logística múltipla.

\begin{tabular}{|c|c|c|c|c|}
\hline Fator avaliado & $\begin{array}{c}\text { Não ajustada } \\
\text { Odds }(95,0 \% \text { IC) }\end{array}$ & p-valor & $\begin{array}{c}\text { Ajustada } \\
\text { Odds }(95,0 \% \text { IC })\end{array}$ & p-valor \\
\hline \multicolumn{5}{|l|}{ Sexo } \\
\hline Feminino & $1,21(0,92-1,59)$ & 0,181 & $1,14(0,86-1,50)$ & 0,374 \\
\hline Masculino & 1 & & 1 & \\
\hline \multicolumn{5}{|l|}{ Idade } \\
\hline$\geq 16$ anos & $1,67(1,27-2,20)$ & $0,000^{\mathrm{a}}$ & $1,64(1,24-2,16)$ & $0,001^{\mathrm{a}}$ \\
\hline$<16$ anos & 1 & & 1 & \\
\hline \multicolumn{5}{|c|}{ Utilização de serviços odontológicos } \\
\hline Regular & $1,35(1,02-1,77)$ & $0,033^{\mathrm{a}}$ & $1,26(0,95-1,66)$ & 0,110 \\
\hline Irregular & 1 & & 1 & \\
\hline \multicolumn{5}{|l|}{ Frequência de escovação dentária } \\
\hline Regular & $6,14(1,47-25,62)$ & $0,013^{\mathrm{a}}$ & $5,56(1,33-23,36)$ & $0,019^{\mathrm{a}}$ \\
\hline Irregular & 1 & & 1 & \\
\hline
\end{tabular}

a Se p-valor $<0,05$ a autoestima influencia nos hábitos de saúde bucal.

Os desafios biopsicossocais vividos pelos adolescentes têm resultados positivos ou negativos que influenciam no nível de autoestima ${ }^{24,34}$. No nosso estudo, foram encontrados resultados semelhantes aos encontrados em escolares da Nova Zelândia ${ }^{23}$, nos quais a menor proporção dos participantes manifestou alta autoestima, e alertam como a adolescência pode ameaçar o nível desse fator psicossocial.

Verificamos que os escolares maiores de 16 anos de idade apresentaram alto nível de autoestima quando comparados aos adolescentes mais jovens. Esse resultado se aproxima aos encontrados em estudos longitudinais, que apontam evolução positiva da autoestima entre a adolescência e a vida adulta, tornando-se estável com o passar dos anos ${ }^{34,35}$. Tal achado ratifica a hipótese de formação da autoestima de Rosenberg ${ }^{32}$, a qual demonstra que esse sentimento é produto das transições oriundas do desenvolvimento humano e evidencia a primordialidade de trabalhar com maior empenho a autoestima em sujeitos mais novos.

Outros fatores sociodemográficos têm sido associados aos níveis de autoestima. Embora tenhamos encontrado que dentre os adolescentes com alto nível de autoestima a maior proporção era do sexo feminino, estudos levantados não apontam para uma análise conclusiva. A desigualdade do nível de autoestima entre os sexos pode estar relacionada à diferença da fonte primária de autoestima, em que as mulheres são mais influenciadas pelas relações sociais e, os homens, pelo êxito em objetivos sociais ${ }^{26,34,35}$.
Também não encontramos relação significativa entre autoestima e as relações escolares e familiares, o que provoca o questionamento do real efeito desses relacionamentos na autoestima, já que existem evidências de repercussão positiva quando os jovens estão inseridos em uma família estruturada ${ }^{37}$, existe apego e apoio dos pais $^{38} \mathrm{e}$ não tem histórico de reprovação escolar ${ }^{34}$. No entanto, o desempenho escolar merece atenção especial porque reflete na autoaceitação, confiança e, consequente, desempenho social ${ }^{22}$. Ou seja, acredita-se que a autoestima do adolescente é apoiada no feedback de pessoas importantes e, quando esses relacionamentos aumentam a autoestima, elevam também o sentimento de competência de enfrentamento dos acontecimentos, como seus problemas de saúde bucal ${ }^{39}$.

Durante a investigação da modulação da autoestima sobre os comportamentos de saúde bucal, foi possível identificar que esse fator psicossocial interfere significativamente nos comportamentos positivos de escovação dentária. Nesta pesquisa, adolescentes com alto nível de autoestima relataram escovar seus dentes com maior frequência. Relação equivalente foi encontrada por Kneckt et al. ${ }^{40}$ ao verificarem que a autoestima pode determinar a aderência aos comportamentos de saúde bucal. Como também, Honkala et al. ${ }^{15}$ acreditam que a ausência de preocupação com a aparência pessoal é a explicação para a clara redução da escovação dentária entre os escolares kuwaitianos, que mencionaram sentimentos de infelicidade, solidão, exclusão social e dificuldades de relacionamento. 
Também foi possível verificar, através da regressão, que a importância da utilização do serviço odontológico diminuiu em relação ao período da adolescência e ao hábito saudável de higiene bucal. Esse achado pode ser justificado pela relação inversa entre aqueles que possuem melhores hábitos de saúde bucal e os que procuram os serviços odontológicos com mais frequência.

Ainda, reconhecendo a existência de um maior risco para saúde bucal entre pessoas com autoestima instável, esse sentimento também não influenciou a frequência da consulta odontológica e a razão principal dessa consulta, dos estudantes romenos interrogados por Dumitrescu et al. ${ }^{26}$. O mesmo aconteceu quando esses mesmos autores tentaram encontrar associação desses comportamentos com o autocontrole e a autoconfiança ${ }^{16}$.

Outros comportamentos estudados, comuns na adolescência e que chamaram nossa atenção, foram o consumo de alimentos não favoráveis à saúde bucal, fumo e bebidas alcoólicas. Apesar de estar bem fundamentado na literatura que esses hábitos manifestam riscos à saúde, incluindo a bucal $^{41,42}$, o poder dos fatores psicossocias sobre eles ainda é divergente. Nossos resultados não evidenciam associações entre a autoestima e o consumo de cigarro e álcool. No entanto, existem estudos que relacionam o sentimento de estigmatização dos fumantes aos baixos níveis de autoestima ${ }^{43,44}$, e outros apontam a alta autoestima como fator protetivo do fumo ${ }^{26,34}$.

Em outro estudo o álcool parece ter papel semelhante ao tabaco. Enquanto jovens com baixa autoestima relataram beber mais para escapar da infelicidade, os que tinham autoestima elevada justificaram o maior consumo de bebida alcoólica pela necessidade de minimizar sua vulnerabilidade entre o grupo social, durante os momentos de recreação ${ }^{44}$. A ausência de associações claras neste estudo, talvez esteja relacionada com o envolvimento de diferentes fenômenos agrupados e com a inconfidência do jovem ao expor suas atitudes de risco, geralmente marginalizadas pela população.

A infidelidade das respostas não deve ter se repetido no relato dos hábitos alimentares, uma vez que a maior proporção dos adolescentes que participaram dessa pesquisa admitiu o consumo de alimentos não favoráveis à saúde bucal em suas dietas, independente do grau de autoestima. Contudo, essa associação talvez precise ser melhor investigada, através de uma medida mias específica, já que ela é oriunda de uma variável de contagem. Como também, cabe atenção para as motivações alimentares desses jovens, dado que a relação da autoestima com os distúrbios alimentares é mu- ito consistente. Por exemplo, dentre os escolares suecos, a autoestima elevada interferiu positivamente em escolhas alimentares saudáveis ${ }^{39}$.

A interpretação de nossos resultados deve levar em consideração a limitação metodológica que a amostra não é representativa da população em geral. Por se tratar de um estudo de base escolar, realizado na rede pública, a universalização dos resultados para os adolescentes de 14 a 19 anos de idade fica comprometida. Portanto, a possibilidade de generalizar os achados do presente estudo deve ser testada, replicando-os em outras populações.

Apesar disso, essa metodologia tem sido adotada em inúmeras pesquisas, pela facilidade em acessar esse grupo populacional e pelos benefícios decorrentes do estudo, o que permite o planejamento integrado dos setores da saúde e educação junto ao público-alvo.

Acredita-se que as evidências apresentadas neste estudo podem auxiliar na identificação de subgrupos mais vulneráveis e, consequentemente, na tomada de decisões e no planejamento de estratégias de intervenção adequadas. Ademais, podem suscitar o desenvolvimento de outras investigações.

Assim, as nossas constatações enfatizam a necessidade de estudos de intervenção que busquem melhorar o sentimento de autoestima em adolescentes. Dessa maneira, as informações geradas favorecerão consideravelmente a compreensão da influência desse fator psicossocial na saúde bucal, e servirão de apoio, para profissionais odontológicos e não odontológicos, nas suas decisões, concepção e desenvolvimento de protocolos para uma efetiva promoção de saúde entre os jovens.

Além disso, nossos achados despertam questões que podem ser esclarecidas em estudos futuros. Para eles, recomendamos pesquisar as consequências da modulação da autoestima nos comportamentos de saúde bucal; e, os possíveis fatores envolvidos, como os diferentes domínios do apoio social, a percepção corporal, a valorização da estética, os fatores socioeconômicos e as atividades de lazer e emprego.

Essas sugestões podem ter melhores dimensões se incluídas em estudos de acompanhamento longitudinal, para que se possa conhecer com mais afinco o impacto desses fatores durante as diferentes faixas etárias, e assim, planejar ações específicas diante as necessidades de cada grupo, melhorando a atenção prestada e a qualidade de vida da população.

Os resultados deste estudo confirmam a modulação da autoestima sobre os comportamentos de saúde bucal de adolescentes e validam nosso 
modelo. Diante de uma população socioeconomicamente homogênea, independente do sexo, escolares maiores de 16 anos e com a autoestima elevada, escovam seus dentes com maior frequência, adquirindo, possivelmente, melhor saúde bucal.

Dessa maneira, enfatizamos a importância dos fatores psicossociais como mediadores de saúde bucal, sendo necessário elevar a autoestima dos jovens para melhorar seus comportamentos de saúde bucal. O que torna a análise e o exercício dos determinantes psicossociais na saúde bucal dos adolescentes partes imprescindíveis para promoção e prevenção de saúde efetivas.

\section{Referências}

1. Marmot MG, Smith GD, Stansfeld S, Patel C, North F, Head J, White I, Brunner E, Feeney A. Health inequalities among British civil servants: the Whitehall II study. Lancet 1991;337(8754):1387-1393.

2. Bosma H, Marmot MG, Hemingway H, Nicholson AG, Brunner E, Stansfeld A. Low job control and risk of coronary heart disease in Whitehall II (prospective cohort) study. BMJ 1997;314(7080):558-565.

3. Marmot MG, Bosma H, Hemingway H, Brunner E, Stansfeld S. Contribution of job control and other risk factors to social variations in coronary heart disease incidence. Lancet 1997;350(9073):235-239.

4. North FM, Syme LS, Feeney A, Shipley M, Marmot M. Psychosocial Work Environment and Sickness Absence among British Civil Servants: The Whitehall II Study. Am J Public Health 1996;86(3):332-340.

5. World Health Organization (WHO). Inequalities young people's health: key findings from the Health Behaviour in School-aged Children (HBSC) 2005/2006 survey fact sheet. Copenhagen: WHO; 2008.

6. Zwig JM, Phillips SD, Lindberg LD. Predicting adolescent profiles of risk: looking beyond demographics. $J$ Adolesc Health 2002;31(4):343-353.

7. World Health Organization (WHO). Growing up unequal: gender and socioeconomic differences in young people's health and well-being: health behaviour in school-aged children (HBSC) study: international report from the 2013/2014 survey. Copenhagen: WHO; 2016.

8. Instituto Brasileiro de Geografia e Estatística (IBGE). Pesquisa Nacional de Saúde do Escolar 2012 [Internet]. Rio de Janeiro: IBGE; 2013. [acessado 2017 Dez 28] Disponível em: https://biblioteca.ibge.gov.br/visualizacao/livros/liv64436.pdf

\section{Colaboradores}

CTC Pazos e PSA Goes realizaram a concepção e desenho da pesquisa, construção do projeto, análise estatística dos dados e interpretação dos resultados, além da redação e revisão do manuscrito. SC Austregésilo realizou análise estatística dos dados e interpretação dos resultados, além da redação e revisão do manuscrito.
9. Malta DC, Sardinha LMV, Mendes I, Barreto SM, Giatti L, Castro IRRD, Moura LD, Dias AJR, Crespo C. Prevalence of risk health behavior among adolescents: Results from the 2009 national adolescent school-based health survey (PeNSE). Ciên Saude Colet 2010;15(2):3009-3019.

10. Ponczek D, Olszowy I. The lifestyle of youth and its impact on health. Probl Hig Epidemiol 2012;93(2):260 268.

11. Yazdani R, Vehkalahti MM, Nouri M, Murtomaa H. Smoking, tooth brushing and oral cleanliness among 15-year-olds in Tehran, Iran. Oral Health Prev Dent 2008;6(1):45-51.

12. Garcia DM, Mekitarian Filho E, Gilio AE, Lotufo JPB, Lo DS. Nutritional status, nutritional self-perception, and use of licit drugs in adolescents. Rev Paul Pediatr 2015;33(3):332-339.

13. Frómeta MM, Pimentel BFT, Silverio BM, Fernández IG, Toledo DT, Rodríguez EH. Labor extensionista desde la universidad médica para prevenir el tabaquismo en niños y adolescentes. Edumecentro 2016;8(1):84-95.

14. Nagaland T, Kadanakuppe S, Raju R. Adolescent's Oral Health-A Review. IJHSR 2016;6(9):420-425.

15. Honkala S, Honkala E, Al-Sahli N. Do life-or school-satisfaction and self-esteem indicators explain the oral hygiene habits of schoolchildren? Community Dent Oral Epidemiol 2007;35(5):337-347.

16. Dumitrescu AL, Dogaru BC, Dogaru CD. Self-control and self-confidence: their relationship to self-rated oral health status and behaviours. Oral Health Prev Dent 2009;7(2):155-162.

17. Dorri M, Sheiham A, Watt RG. Modelling the factors influencing general and oral hygiene behaviours in adolescents. Int J Paediatr Dent 2010;20(4):261-269. 
18. Silva AN, Mendonça MH, Vettore MV. The association between low-socioeconomic status mother's Sense of Coerence and their child's utilization of dental care. Community Dent Oral Epidemiol 2011;39(2):115-126.

19. Carvalho PDD, Barros MVGD, Lima RA, Santos CM, Mélo EN. Condutas de risco à saúde e indicadores de estresse psicossocial em adolescentes estudantes do Ensino Médio. Cad Saude Publica 2011;27(11):20952105.

20. Baker SR, Mat A, Robinson PG. What Psychosocial Factors Influence Adolescents' Oral Health? J Dent Res 2010;89(11):1230-1235.

21. Agou S, Locker D, Muirhead V, Tompson B, Streiner DL. Does psychological well-being influence oral-health-related quality of life reports in children receiving orthodontic treatment? Am J Orthod Dentofacial Orthop 2011;139(3):369-377.

22. Bandeira CDM, Hutz CS. As implicações do bullying na auto-estima de adolescentes. Psicologia Escolar e Educacional 2010;14(1):131-138.

23. Foster Page LA, Thomson WM, Ukra A, Baker SR. Clinical status in adolescents: is its impact on oral health-related quality of life influenced by psychological characteristics? Eur J Oral Sci 2013;121(3pt1):182187.

24. Källestål C, Dahlgren L, Stenlund H. Oral health behavior and self-esteem in Swedish adolescents over four years. J Adolesc Health 2006;38(5):583-590.

25. Agou S, Locker D, Streiner DL, Tompson B. Impact of self-esteem on the oral-health-related quality of life of children with malocclusion. Am J Orthod Dentofacial Orthop 2008;134(4):484-489.

26. Dumitrescu AL, Dogaru CB, Dogaru CD. Instability of Self-esteem and Affective Lability as Determinants of Self-reported Oral Health Status and Oral Health-related Behaviors. I Contemp Dent Pract 2008;9(1):38-45.

27. Instituto Brasileiro de Geografia e Estatística (IBGE). Pesquisa nacional por amostra de domicílios 2010 [Internet]. Rio de Janeiro: IBGE; 2011. [acessado 2017 Dez 28] Disponível em: http://www.cidades.ibge.gov. br/xtras/perfil.php?lang $=\&$

28. Brasil. Ministério da Saúde (MS). Secretaria de Atenção à Saúde/Secretaria de Vigilância em Saúde. Departamento de Atenção Básica. Coordenação Geral de Saúde Bucal. SB Brasil 2010 - Resultados Principais. Brasília: MS; 2011.

29. Bezerra IA, Goes PSA. Associação entre capital social, condições e comportamentos de saúde bucal. Ciên Saude Colet 2014;19(6):1943-1950.

30. Kozmhinsky VMR, Heimer M, Goes PSA. Sociodemographic factors and oral health conditions related to the impact on the quality of life of adolescents. Pesqui Bras Odontopediatria Clin Integr 2016;16(1):35-42.

31. Instituto Nacional de Estudos e Pesquisas Educacionais Anísio Teixeira (INEP). Sinopse estatística da educação básica 2014 [Internet]. Brasília: INEP; 2016. [acessado 2018 Jan 05] Disponível em: http://portal. inep.gov.br/sinopses-estatisticas-da-educacao-basica

32. Rosenberg M. Society and the adolescent self-image. New Jersey: Princeton University Press; 1965.
33. Sbicigo JB, Bandeira DR, Dell'aglio DD. Escala de Autoestima de Rosenberg (EAR): validade fatorial e consistência interna. Rev Psico-USF 2010;15(3):395-403.

34. Kiviruusu O, Huurre T, Aro H, Marttunen M, Haukkala A. Self-esteem growth trajectory from adolescence to mid-adulthood and its predictors in adolescence. Advances in Life Course Research 2015; 23:29-43.

35. Erol RY, Orth U. Self-esteem development from age 14 to 30 years: a longitudinal study. J Abnorm Soc Psychol 2011;101(3):607.

36. Menezes RCE, Osório MM. Consumo energético-proteico e estado nutricional de crianças menores de cinco anos, no estado de Pernambuco, Brasil. Revista de Nutrição 2007;20(4):337-347.

37. D'onofrio BM, Turkheimer E, Emery RE, Slutske WS, Heath AC, Madden PA, Martin NG. A genetically informed study of the processes underlying the association between parental marital instability and offspring adjustment. Dev Psychol 2006;42(3):486-499.

38. Kang S, Jeon H, Kwon S, Park S. Parental attachment as a mediator between parental social support and self-esteem as perceived by korean sports middle and high school athletes. Percept Mot Skills 2015;120(1):288-303.

39. Källestål C, Dahlgren L, Stenlund H. Oral health behaviour and self-esteem in Swedish children. Soc Sci Med 2000;51(12):1841-1849.

40. Kneckt MC, Keinänen-Kiukaanniemi SM, Knuuttila ML, Syrjälä AMH. Self-esteem as a characteristic of adherence to diabetes and dental self-care regimens. J Clin Periodontol 2001;28(2):175-180.

41. Watt RG. Strategies and approaches in oral disease prevention and health promotion. Bull World Health Organ 2005;83(9):711-718.

42. Watt RG, Harnett R, Daly B, Fuller SS, Kay E, Morgan A, Munday P, Nowjack-Raymer R, Treasure ET. Evaluating oral health promotion: need for quality outcome measures. Community Dent Oral Epidemiol 2006;34(1):11-17.

43. Ariza C, Nebot M, Villalbí JR, Díez E, Tomás Z, Valmayor $\mathrm{S}$. Tendencias en el consumo de tabaco, alcohol y cannabis de los escolares de Barcelona (1987-1999). Gac Sanit 2003;17(3):190-195.

44. Baumeister RF, Campbell JD, Krueger JI, Vohs KD. Does high self-esteem cause better performance, interpersonal success, happiness, or healthier lifestyles? Psychol Sci Public Interest 2003;4(1):1-44.

Artigo apresentado em 22/09/2017

Aprovado em 24/04/2018

Versão final apresentada em 26/04/2018 